\title{
Convergence of lattice sums and Madelung's constant
}

\author{
David Borwein \\ Department of Mathematics, University of Western Ontario, London, Ontario, N6A 5B9, Canada
}

Jonathan M. Borwein

Department of Mathematics, Statistics and Computing Science, Dalhousie University, Halifax, Nova Scotia, B3H 4H8, Canada

Keith F. Taylor

Department of Mathematics, University of Saskatchewan, Saskatoon, Saskatchewan, S7N OWO, Canada

(Received 13 September 1984; accepted for publication 3 June 1985)

\begin{abstract}
The lattice sums involved in the definition of Madelung's constant of an NaCl-type crystal lattice in two or three dimensions are investigated. The fundamental mathematical questions of convergence and uniqueness of the sum of these, not absolutely convergent, series are considered. It is shown that some of the simplest direct sum methods converge and some do not converge. In particular, the very common method of expressing Madelung's constant by a series obtained from expanding spheres does not converge. The concept of analytic continuation of a complex function to provide a basis for an unambiguous mathematical definition of Madelung's constant is introduced. By these means, the simple intuitive direct sum methods and the powerful integral transformation methods, which are based on theta function identities and the Mellin transform, are brought together. A brief analysis of a hexagonal lattice is also given.
\end{abstract}

\section{INTRODUCTION}

Lattice sums have played a role in physics for many years and have received a great deal of attention on both practical and abstract levels. The term lattice sum is not a precisely defined concept: it refers generally to the addition of the elements of an infinite set of real numbers, which are indexed by the points of some lattice in $N$-dimensional space. A method of performing a lattice sum involves accumulating the contributions of all these elements in some sequential order. Unfortunately, the elements of the set are not, in general, absolutely summable so the sequential order chosen can affect the answer. In this paper we are concerned with the particular lattice sums involved in Madelung's constant. Indeed, attaining specificity in the definition of Madelung's constant is our primary motive. Although we are dealing with purely mathematical questions it is our belief that the results presented here may shed some light on the physics of crystals. Other researchers ${ }^{1-3}$ have expressed concern about the ambiguities involved in summing a nonabsolutely convergent series in a different manner, but it appears that no one has confronted it fully.

Let $L$ be a lattice in $N$-dimensional space and let $A_{L}=\left\{a_{1}: l \in L\right\}$ be a set of real numbers indexed by $L$. There are two basic approaches to summing the elements of $A_{L}:$ by direct summation or by integral transformations. The major factors involved in choosing a method are physical meaningfulness and speed of convergence.

The direct summation methods involve an orderly grouping of the elements of $A_{L}$ into sequentially indexed finite subsets increasing in size to eventually include any element of $A_{L}$. Sometimes fractions of elements are included in the subsets to maintain a physical principal such as electrical neutrality. Two commonly used direct summation methods are due to Evjen ${ }^{4}$ and Højendahl. ${ }^{5}$
The most commonly used integral transformation method is known as the Ewald method. ${ }^{6}$ More recently the Mellin transformation applied to theta functions has been used to put the integral transformation methods in a general context. An excellent review article by Glasser and Zucker ${ }^{1}$ gives a development of these methods and an extensive bibliography.

In this paper we deal primarily with $\mathrm{NaCl}$-type ionic crystals in two or three dimensions. This is for two main reasons: the ease of notation and the fact that almost every textbook introduces Madelung's constant on this crystal first. From a mathematical and physical point of view there are two very reasonable simple direct summation methods that could be applied to give Madelung's constant for an $\mathrm{NaCl}$-type ionic crystal. One could take a basic cube centered at the referenced ion with sides parallel to the basic vectors and let the cube expand as the contributions from all lattice points within the cube are accumulated. Alternately one could use expanding spheres centered at the reference ion. This latter method is intuitively appealing since all ions an equal distance from the reference ion are given equal treatment. Thus, many textbooks ${ }^{7,8}$ (and some research articles) write down the resulting infinite series $(6-12 / \sqrt{2}+8 /$ $\sqrt{3}-6 / \sqrt{4}+\cdots)$ as giving Madelung's constant for an $\mathrm{NaCl}-$ type ionic crystal. Unfortunately, this infinite series does not converge. This was proven by Emersleben' and, in light of the fact that most people are unaware of this divergence, we include a short elementary proof in Theorem 3.

Section II is devoted to the two-dimensional square lattice while Sec. III contains the above-mentioned result on expanding spheres. In Theorem 4 we prove that the method of expanding cubes converges. In Sec. IV, the mathematical tools become more sophisticated as we consider integral transformation methods and their relation to the direct sum- 
mation methods dealt with in Sec. III. We have included, in Sec. V, a careful analysis of some direct summation methods in two dimensions in light of the property of being analytic in the inverse power exponent. This analysis is quite illustrative of the relations between the various summation methods. In Sec. VI we do a brief analysis of a two-dimensional hexagonal lattice. Section VII gives our conclusions.

\section{TWO DIMENSIONS}

It is convenient to introduce the notation in the twodimensional case of a simple lattice in the plane with unit charges located at integer lattice points $(j, k)$ and of sign $(-1)^{j+k}$. The potential energy at the origin due to the charge at $(j, k)$ is $-(-1)^{j+k} /\left(j^{2}+k^{2}\right)^{1 / 2}$. If we want the total potential energy at the origin due to all other charges, then we must sum all the numbers in the following set:

$$
A=\left\{(-1)^{j+k} /\left(j^{2}+k^{2}\right)^{1 / 2}:(j, k) \in \mathbf{Z} /(0,0)\right\},
$$

where $\mathbf{Z}$ denotes the integers. Because the elements of the subset of $A$ with $j=k$ form a set of positive numbers with divergent sum, it is clear that the value of the sum is highly dependent on the order in which the elements of $A$ are added. It is not immediately clear that any reasonable method will produce a convergent series. In addition, for the model to be physically relevant, all "reasonable" methods should converge to the same number. Here are two very reasonable methods.

First, consider the total potential due to all the charges within a circle of radius $r$ about the origin and let $r \rightarrow \infty$. This leads to the series

$$
\sum_{n=1}^{\infty} \frac{(-1)^{n} C_{2}(n)}{n^{1 / 2}}
$$

where $C_{2}(n)$ is the number of ways of writing $n$ as a sum of two squares of integers (positive, negative, or zero). In deriving (1), use the fact that $(-1)^{j+k}=(-1)^{f+k^{2}}=(-1)^{n}$, for any $j, k \in Z$ with $j^{2}+k^{2}=n$. We will refer to (1) as the method of expanding circles.

Second, there is the method of expanding squares. This is intuitively appealing, as a perfect crystal grows by expansion of the shape of a basic unit cell. For each natural number $n$, let

$S_{2}(n)=\sum\left\{\frac{(-1)^{j+k}}{\left(j^{2}+k^{2}\right)^{1 / 2}}:-n<j, k<n\right.$ and $\left.(j, k) \neq(0,0)\right\}$.

Then

$$
\lim _{n \rightarrow \infty} S_{2}(n)
$$

is a way of expressing the series obtained by expanding squares.

It turns out that both these methods converge as we will now show.

Theorem 1: The series in (1) converges.

Proof: To carry out the proof that the series in (1) converges we introduce some notation and standard facts from number theory. For any sequence of real numbers $\left\{a_{n}\right\}_{n=1}^{\infty}$ and $\beta$ real we write $a_{n}=O\left(n^{\beta}\right)$ if the sequence $\left\{n^{-\beta} a_{n}\right\}$ is bounded. Let $A_{n}=\sum_{k=1}^{n^{\prime}} C_{2}(k)$, for each natural number $n$. Then $A_{n}$ denotes the number of nonorigin lattice points inside or on a circle of radius $n^{1 / 2}$. It is fairly easy to see that $A_{n}$ should be approximately $\pi n$; in fact, the reader can easily show that $A_{n}-\pi n=O\left(n^{1 / 2}\right)$. However, this is not quite good enough for us here so we quote a stronger result, which can be found in Dickson ${ }^{10}$ :

$$
A_{n}-\pi n=O\left(n^{\alpha}\right), \quad \text { for some } \alpha, \quad \frac{1}{4}<\alpha<\frac{1}{3} \text {. }
$$

For a natural number $n$, a divisor of $n$ is a natural number $d$ such that $d$ divides $n$. Let $d(n)$ denote the number of divisors of $n$ and let $d_{k}(n)$ denote the number of divisors $d$ of $n$ with $d$ congruent to $k$ modulo 4 , for $k=1$ or 3 . With this notation, Theorem 278 of Hardy and Wright ${ }^{11}$ implies

$$
C_{2}(n)=4\left(d_{1}(n)-d_{3}(n)\right) \text {. }
$$

This together with Theorem 315 of Hardy and Wright ${ }^{11}$ implies that

$$
C_{2}(n)=O\left(n^{\delta}\right), \quad \text { for any } \delta>0 .
$$

Note that, $d_{k}(2 n)=d_{k}(n)$, for $k=1$ or 3 and any $n$. So

$$
C_{2}(2 n)=C_{2}(n) \text {, for all natural numbers } n \text {. }
$$

Let $B_{n}=\Sigma_{k=1}^{n}(-1)^{k} C_{2}(k)$. Then

$$
\begin{aligned}
B_{2 n} & =\sum_{k=1}^{2 n}(-1)^{k} C_{2}(k) \\
& =\sum_{k=1}^{n} C_{2}(2 k)-\sum_{k=1}^{n} C_{2}(2 k-1) \\
& =2 \sum_{k=1}^{n} C_{2}(2 k)-\sum_{k=1}^{2 n} C_{2}(k) .
\end{aligned}
$$

Using (6),

$$
B_{2 n}=2 A_{n}-A_{2 n} \text {. }
$$

From (3) and (7), we have that, with $\alpha$ as in (3),

$$
B_{2 n}=O\left(n^{\alpha}\right) \text {. }
$$

Furthermore, this along with (5) implies that

$$
B_{2 n+1}=B_{2 n}-C_{2}(2 n+1)=O\left(n^{\alpha}\right)
$$

Therefore,

$$
B_{n}=O\left(n^{\alpha}\right) \text {. }
$$

Now consider the partial sums of the series in (1):

$$
\begin{aligned}
T_{n}= & \sum_{k=1}^{n} \frac{(-1)^{k} C_{2}(k)}{k^{1 / 2}}=\frac{B_{n}}{(n+1)^{1 / 2}} \\
& +\sum_{k=1}^{n} B_{k}\left[k^{-1 / 2}-(k+1)^{-1 / 2}\right] \\
= & O\left(n^{\alpha-1 / 2}\right)-\sum_{k=1}^{n} B_{k}\left[(k+1)^{-1 / 2}-k^{-1 / 2}\right] .
\end{aligned}
$$

By the mean value theorem, $\left|(k+1)^{-1 / 2}-k^{-1 / 2}\right|<\frac{1}{2} k^{-3 / 2}$ and therefore

$$
\left|B_{k}\left[(k+1)^{-1 / 2}-k^{-1 / 2}\right]\right|=O\left(k^{\alpha-3 / 2}\right) .
$$

Since $\alpha-3 / 2<-1, \sum_{k=1}^{\infty} B_{k}\left[(k+1)^{-1 / 2}-k^{-1 / 2}\right]$ converges absolutely. Since $\alpha-\frac{1}{2}<0, \lim _{n \rightarrow \infty} T_{n}$ $=-\sum_{k=1}^{\infty} B_{k}\left[(k+1)^{-1 / 2}-k^{-1 / 2}\right]$ exists. That is, the series in (1) converges.

Q.E.D.

We now turn to the limit in (2). We need an easy lemma from calculus that will be left to the reader to verify. This lemma will also be used in the proof of Theorem 4. 
Lemma: For any positive real numbers, $a, b$, and $s$, each of the following functions are strictly decreasing in $t$, for $0<t<\infty$ :

$$
\begin{aligned}
f_{1, s}(t) & =t^{-s}, \\
f_{2, s}(t) & =t^{-s}-(t+a)^{-s}, \\
f_{3, s}(t) & =t^{-s}-(t+a)^{-s} \\
& \quad-(t+b)^{-s}+(t+a+b)^{-s} .
\end{aligned}
$$

Theorem 2: The limit in (2) exists.

Proof: We apply the lemma to $f_{2,5}$ with $a=(k+1)^{2}-k^{2}$ and $s=\frac{1}{2}$. Then, if $j>0$ and $k \geqslant 0$ with $j+k>1$, we have

$$
\begin{aligned}
& f_{2,1 / 2}\left(j^{2}+k^{2}\right)>f_{2,1 / 2}\left((j+1)^{2}+k^{2}\right) . \\
& \text { Explicitly this is } \\
& \left(j^{2}+k^{2}\right)^{-1 / 2}-\left(j^{2}+(k+1)^{2}\right)^{-1 / 2} \\
& -\left((j+1)^{2}+k^{2}\right)^{-1 / 2}+\left((j+1)^{2}+(k+1)^{2}\right)^{-1 / 2}>0 .
\end{aligned}
$$

Let $g(j, k)$ denote the left-hand side of $(10)$. Then $(-1)^{j+k}$ $X g(j, k)$ is the contribution to the potential at the origin due to a basic cell of four adjacent ions with the closest ion at $(j, k)$. Inequality $(10)$ says that the contribution always has the same sign as that of the nearest ion.

Rewrite $S_{2}(n)$ using the symmetries to get

$$
S_{2}(n)=4 Q(n)+4 X(n) \text {, }
$$

where

$$
\begin{aligned}
& Q(n)=\sum_{j, k=1}^{n} \frac{(-1)^{j+k}}{\left(j^{2}+k^{2}\right)^{1 / 2}}, \\
& X(n)=\sum_{k=1}^{n} \frac{(-1)^{k}}{k} .
\end{aligned}
$$

Since $\lim _{n \rightarrow \infty} X(n)=-\ln 2$, if we prove that $\lim _{n \rightarrow \infty} Q(n)$ exists, then the limit in (2) will exist. We will establish a number of properties of the sequence $\{Q(n)\}_{n=1}^{\infty}$, which will be used to prove its convergence.

\section{Property 1:}

$$
Q(2 n)-Q(2 n-2)>0, \text { for all } n>2 \text {. }
$$

That is, the even indexed elements increase. To see this we group the terms of $Q(2 n)-Q(2 n-2)$ into basic cells of 4 , as is illustrated in Fig. 1(a) for $Q(6)-Q(4)$. Thus,

$$
\begin{aligned}
& Q(2 n)-Q(2 n-2) \\
& =\sum_{l=1}^{n} g(2 l-1,2 n-1)+\sum_{m=1}^{n-1} g(2 n-1,2 m-1),
\end{aligned}
$$

where as before, $g(j, k)$ denotes the left-hand side of $(10)$. So property 1 holds.

Property 2:

$$
Q(2 n+1)-Q(2 n-1)<0, \text { for all } n>1 \text {. }
$$

That is, the odd indexed elements of the sequence decreases. Referring to Fig. 1(b) and correcting for the overlap at the $(2 n, 2 n)$ point we are led to the following grouping:

$$
\begin{aligned}
& Q(2 n+1)-Q(2 n-1) \\
& \quad=\sum_{l=1}^{n}[-g(2 l-1,2 n)]+\sum_{l=1}^{n}[-g(2 n, 2 l-1)] \\
& -[1 / n \sqrt{2}-1 /(n+1) \sqrt{2}]<0 .
\end{aligned}
$$

\section{Property 3:}

$$
Q(2 n+1)-Q(2 n)>0, \text { for all } n>1 \text {. }
$$

Thus, the odd indexed elements are all greater than any even indexed element. This is clear again from a simple grouping of terms and using the monotonicity of $f_{1,1 / 2}$ of the lemma:

$$
\begin{aligned}
& Q(2 n+1)-Q(2 n) \\
&=2 \sum_{l=1}^{n}\left[\frac{1}{\left((2 l-1)^{2}+(2 n+1)^{2}\right)^{1 / 2}}\right. \\
&\left.-\frac{1}{\left(4 l^{2}+(2 n+1)^{2}\right)^{1 / 2}}\right]+\frac{1}{(2 n+1) \sqrt{2}}>0 .
\end{aligned}
$$

\section{Property 4:}

$$
\lim _{n \rightarrow \infty} Q(2 n-1)-Q(2 n)=0 .
$$

Thus, the difference between successive elements goes to zero. To see this, simply note that

$$
\begin{aligned}
0< & Q(2 n+1)-Q(2 n)<2 /\left[1+(2 n+1)^{2}\right]^{1 / 2} \\
& +1 /(2 n+1) \sqrt{2} \rightarrow 0, \text { as } n \rightarrow \infty .
\end{aligned}
$$

It is now easy to see that properties $1-4$ imply that $\lim _{n \rightarrow \infty}$ $Q(n)$ exists.

Q.E.D.

This completes the proof of Theorem 2. Thus, we have shown that two of the most obvious methods of summing for a Madelung constant in two dimensions converge. At this point, no indication has been given that the two methods yield the same number. That this is indeed so will be shown in Sec. $V$.

\section{THREE DIMENSIONS}

In this section, the three-dimensional case will be considered. For Madelung's constant of an NaCl-type crystal one must investigate ways of summing the elements of the following set:

$$
\begin{aligned}
B= & \left\{(-1)^{j+k+l} /\left(j^{2}+k^{2}+l^{2}\right)^{1 / 2}:\right. \\
& \left.(j, k, l) \in \mathbf{Z}^{3} /(0,0,0)\right\} .
\end{aligned}
$$

In analogy with the two-dimensional case we will consider the method of expanding spheres about the origin and the method of expanding cubes.

Our next theorem is a negative result, which is quite startling. Many textbooks in physical chemistry and solid state physics give the series dealt with in Theorem 3 as Madelung's constant for a NaCl-type crystal. ${ }^{7.8}$ It also appears

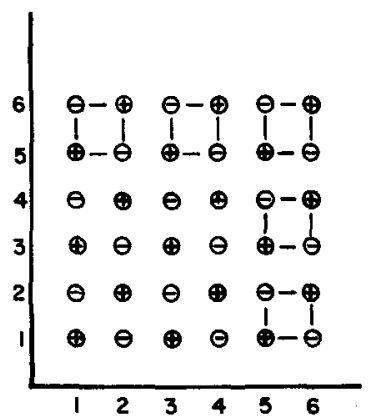

(a)

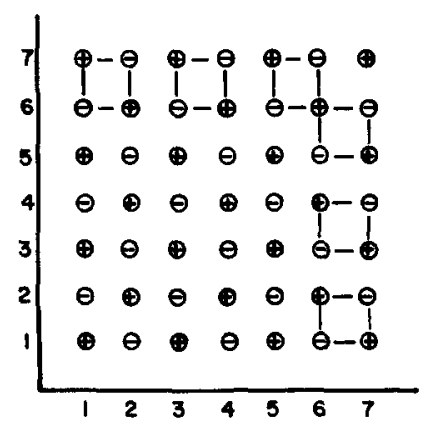

(b)
FIG. 1. Illustrations of (a) property 1 and (b) property 2. 
in research articles. Although no one sums this series directly, it is physically misleading to believe that it converges to anything.

Let $C_{3}(n)$ denote the number of ways of writing $n$ as a sum of three squares. If we consider a sphere centered at the origin in three-space, add all the elements of $B$ that correspond to lattice points within the sphere, and then let the radius go to infinity, we are led to the infinite series

$$
\sum_{n=1}^{\infty} \frac{(-1)^{n} C_{3}(n)}{\sqrt{n}}
$$

Theorem 3 (Emersleben'): The series in (11) diverges.

Proof: It is interesting that the proof that the series in (11) diverges is much less sophisticated than the proof in Theorem 1 that the series in (1) converges. Our main tool is a simple estimate of the number of nonzero lattice points on or inside a sphere of radius $r$. Call this number $L_{r}$. Notice that, for $\sqrt{n}<r<\sqrt{n+1}$,

$$
L_{r}=\sum_{k=1}^{n} C_{3}(k) \text {. }
$$

We leave to the reader the easy task of verifying that

$$
L_{r}-\frac{4}{3} \pi r^{3}=O\left(r^{2}\right) \text {. }
$$

This implies that

$$
\lim _{r \rightarrow \infty} L_{r} / r^{3}=\frac{4}{3} \pi
$$
that

Proceeding with a proof by contradiction we assume

$$
\sum_{n=1}^{\infty} \frac{(-1)^{n} C_{3}(n)}{\sqrt{n}} \text { converges. }
$$

This implies that $\epsilon_{n}=C_{3}(n) / \sqrt{n} \rightarrow 0$, as $n \rightarrow \infty$. For a natural number $N$, let $M_{N}=\max \left\{\epsilon_{n}: n>N\right\}$. Then $M_{N} \rightarrow 0$, as $N \rightarrow \infty$. Fix $N$ for the moment and consider, for $n>N$,

$$
\begin{aligned}
\frac{L_{\sqrt{n}}}{(\sqrt{n})^{3}} & =n^{-3 / 2}\left[\sum_{k=1}^{n} \epsilon_{k} \sqrt{k}\right] \\
& <n^{-3 / 2}\left[\sum_{k=1}^{N} \epsilon_{k} \sqrt{k}\right]+M_{N} n^{-3 / 2}\left[\sum_{k=N+1}^{n} \sqrt{k}\right] .
\end{aligned}
$$

Now,

$$
\begin{aligned}
\sum_{k=N+1}^{n} \sqrt{k} & <\int_{N+1}^{n+1} t^{1 / 2} d t \\
& =\frac{2}{3}\left[(n+1)^{3 / 2}-(N+1)^{3 / 2}\right] .
\end{aligned}
$$

Inserting this in (13) implies that

$$
\begin{aligned}
\frac{L_{\sqrt{n}}}{(\sqrt{n})^{3}}<n^{-3 / 2}\left[\sum_{k=1}^{N} \epsilon_{k} \sqrt{k}\right] \\
\quad+\frac{2}{3} M_{n}\left[\left(\frac{n+1}{n}\right)^{3 / 2}-\left(\frac{N+1}{n}\right)^{3 / 2}\right] .
\end{aligned}
$$

Letting $n \rightarrow \infty$, we see that $\lim \sup _{n \rightarrow \infty} L_{\sqrt{n}} /(\sqrt{n})^{3}<2 M_{N}$, for any $N$. Since $M_{N} \rightarrow 0$ as $N \rightarrow \infty$, we have that

$$
\lim _{n \rightarrow \infty} \frac{L_{\sqrt{n}}}{(\sqrt{n})^{3}}=0 .
$$

This is a contradiction of (12). Therefore,

$$
\sum_{n=1}^{\infty} \frac{(-1)^{n} C_{3}(n)}{\sqrt{n}} \text { diverges. }
$$

In fact, the contributions of individual spherical shells do not tend to zero.

Q.E.D.

So it is not at all appropriate to define Madelung's constant via the method of expanding spheres. We turn to the method of expanding cubes. Let

$$
\begin{aligned}
S_{3}(n)= & \sum\left\{\frac{(-1)^{j+k+l}}{\left(j^{2}+k^{2}+l^{2}\right)^{1 / 2}}:\right. \\
& -n<j, k, l<n,(j, k, l) \neq(0,0,0)\} .
\end{aligned}
$$

Theorem 4: $\lim _{n \rightarrow \infty} S_{3}(n)$ exists.

Proof: We proceed as in the proof of Theorem 2 in Sec. II. For $j, k, l>1$ let

$$
\begin{aligned}
g(j, k, l)= & \left(j^{2}+k^{2}+l^{2}\right)^{-1 / 2}-\left(j^{2}+(k+1)^{2}+l^{2}\right)^{-1 / 2} \\
& -\left(j^{2}+k^{2}+(l+1)^{2}\right)^{-1 / 2} \\
& +\left(j^{2}+(k+1)^{2}+(l+1)^{2}\right)^{-1 / 2} \\
& -\left((j+1)^{2}+k^{2}+l^{2}\right)^{-1 / 2} \\
& +\left((j+1)^{2}+(k+1)^{2}+l^{2}\right)^{-1 / 2} \\
& +\left((j+1)^{2}+k^{2}+(l+1)^{2}\right)^{-1 / 2} \\
& -\left((j+1)^{2}+(k+1)^{2}\right. \\
& \left.+(l+1)^{2}\right)^{-1 / 2}
\end{aligned}
$$

Then, $(-1)^{j+k+l} g(j, k, l)$ represents the contribution to the potential at the origin of a basic unit cell whose closest corner is at $(j, k, l)$. An appropriate use of the monotonicity of $f_{3,1 / 2}$ from the lemma in Sec. II shows that $g(j, k, l)>0$, for all $j, k, l>1$.

Let

$$
\begin{aligned}
h(k, l)= & \left(2 k^{2}+l^{2}\right)^{-1 / 2}-\left(2 k^{2}+(l+1)^{2}\right)^{-1 / 2} \\
& +\left(2(k+1)^{2}+l^{2}\right)^{-1 / 2} \\
& -\left(2(k+1)^{2}+(l+1)^{2}\right)^{-1 / 2} .
\end{aligned}
$$

Using $f_{2,1 / 2}$, we get that $h(k, l)>0$, for all $k, l>1$.

Let $P(n)$ denote that part of $S_{3}(n)$ that comes from the positive octant. That is, for $n>1$,

$$
P(n)=\sum_{j, k, T=1}^{n} \frac{(-1)^{j+k+l}}{\left(j^{2}+k^{2}+l^{2}\right)^{1 / 2}}
$$

Then in a manner similar that used for the $Q(n)$ 's, it can be shown that $\lim _{n \rightarrow \infty} P(n)$ exists. We proceed with the details of this demonstration.

The following identities are most easily seen by drawing a three-dimensional version of Fig. 1, but they can be verified directly:

$$
\begin{aligned}
P(2 n+1)-P(2 n-1)= & 3 \sum_{k, t=1}^{n} g(2 n, 2 k-1,2 l-1) \\
& +3 \sum_{j=1}^{n} h(2 n, 2 j-1) \\
& +(1 / \sqrt{3})(1 / 2 n-1 /(2 n+1)),
\end{aligned}
$$




$$
\begin{aligned}
P(2 n+2)-P(2 n)= & -3 \sum_{k, t=1}^{n} g(2 n+1,2 k-1,2 l-1) \\
& -3 \sum_{j=1}^{n} g(2 n+1,2 n+1,2 j-1) \\
& -g(2 n+1,2 n+1,2 n+1) .
\end{aligned}
$$

Both (14) and (15) hold for all $n>1$. From (14) and (15) we get the properties of odd or even element monotonicity of the sequence of $P(n)$ 's.

Property 1':

$P(2 n)-P(2 n-2)<0$, for all $n>2$.

Property 2':

$P(2 n+1)-P(2 n-1)>0$, for all $n \geq 1$.

Notice that the inequalities are reversed from those of properties 1 and 2 in the two-dimensional case. To get the analogs of properties 3 and 4 for the $P(n)$ 's we need to refer to the lemma one final time. For $n, j, k \geqslant 1$, let

$$
\begin{aligned}
h_{0}(n, j, k)= & \left(n^{2}+j^{2}+k^{2}\right)^{-1 / 2}-\left(n^{2}+(j+1)^{2}+k^{2}\right)^{-1 / 2} \\
& -\left(n^{2}+j^{2}+(k+1)^{2}\right)^{-1 / 2} \\
& +\left(n^{2}+(j+1)^{2}+(k+1)^{2}\right)^{-1 / 2} .
\end{aligned}
$$

With $a=(k+1)^{2}-k^{2}$,

$h_{0}(n, j, k)=f_{2,1 / 2}\left(n^{2}+j^{2}+k^{2}\right)-f_{2,1 / 2}\left(n^{2}+(j+1)^{2}+k^{2}\right)$,

which is positive for all $n, j, k \geqslant 1$.

With this notation

$$
\begin{aligned}
P(2 n+1)-P(2 n)= & -3 \sum_{j, k=1}^{n} h_{0}(2 n+1,2 j-1,2 k-1) \\
& -3 \sum_{l=1}^{n}\left[\left(2(2 n+1)^{2}+(2 l-1)^{2}\right)^{-1 / 2}\right. \\
& \left.-\left(2(2 n+1)^{2}+(2 l)^{2}\right)^{-1 / 2}\right] \\
& -1 /((2 n+1) \sqrt{3}) .
\end{aligned}
$$

This leads to the following property.

Property 3':

$P(2 n+1)-P(2 n)<0$, for all $n>1$.

Therefore, the decreasing even indexed elements are all greater than the increasing odd indexed elements. To see that there is a unique limit to the sequence of the $P(n)$ 's, we only need the last property, which implies that the distance between successive terms approaches zero. This follows from (16) and

$$
\begin{aligned}
P(2 n+1)-P(2 n)> & -3 /\left((2 n+1)^{2}+2\right)^{1 / 2} \\
& -1 /((2 n+1) \sqrt{3}) .
\end{aligned}
$$

To verify (17) let

$$
x_{j}=\sum_{k=1}^{2 n} \frac{(-1)^{1+j+k}}{\left((2 n+1)^{2}+j^{2}+k^{2}\right)^{1 / 2}}, \text { for } 1<j<2 n+1 .
$$

Using the function $h_{0}$, defined above, write

$$
\left|x_{j}\right|-\left|x_{j+1}\right|=\sum_{k=1}^{n} h_{0}(2 n+1, j, 2 k-1)>0 .
$$

Note that $x_{j}$ itself is an alternating sum of decreasing terms, so the sign of $x_{j}$ is $(-1)^{j}$. With (18), this implies that

$$
0>\sum_{j=1}^{2 n+1} x_{j}>x_{1}>\frac{-1}{\left((2 n+1)^{2}+2\right)^{-1 / 2}} \text {. }
$$

Then,

$$
\begin{aligned}
P(2 n+1)-P(2 n)= & 3 \sum_{j=1}^{2 n+1} x_{j}-\frac{1}{(2 n+1) \sqrt{3}} \\
> & -\frac{3}{\left((2 n+1)^{2}+2\right)^{1 / 2}} \\
& -\frac{1}{((2 n+1) \sqrt{3})} .
\end{aligned}
$$

So (17) holds. Thus, the following property has been established.

Property 4':

$$
\lim _{n \rightarrow \infty} P(2 n+1)-P(2 n)=0 .
$$

Properties $1^{\prime}-4^{\prime}$ imply that $\lim _{n \rightarrow \infty} P(n)$ exists.

Finally,

$$
S_{3}(n)=8 P(n)+8 Q(n)+6 X(n),
$$

where, as before,

$$
Q(n)=\sum_{j, k=1}^{n} \frac{(-1)^{j+k}}{\left(j^{2}+k^{2}\right)^{1 / 2}}
$$

and

$$
X(n)=\sum_{k=1}^{n} \frac{(-1)^{k}}{k}
$$

Since each of the terms on the right-hand side of (19) approach a limit as $n \rightarrow \infty$, we have that $\lim _{n \rightarrow \infty} S_{3}(n)$ exists.

Q.E.D.

\begin{tabular}{|c|c|c|c|c|c|c|}
\hline$i \vee j$ & 1 & 2 & 3 & 4 & 5 & \\
\hline 1 & 1 & $-\frac{1}{2}$ & 1 & -1 & $\frac{1}{3}$ & $\cdots$ \\
\hline 2 & $-\frac{1}{2}-10^{-2}$ & $\frac{1}{2}-10^{-3}$ & $-\frac{1}{4}-10^{-4}$ & $t-10^{-5}$ & $-\frac{1}{6}-10^{-6}$ & ... \\
\hline 3 & $\frac{1}{2}$ & $-\frac{1}{3}$ & 1 & $-\frac{1}{3}$ & $\frac{1}{6}$ & ... \\
\hline 4 & $-\frac{1}{3}-10^{-3}$ & $3-10^{-4}$ & $-b-10^{-5}$ & $1-10^{-6}$ & $-1-10^{-7}$ & $\cdots$ \\
\hline 5 & $\frac{1}{3}$ & -1 & 1 & $-\frac{1}{6}$ & 4 & $\cdots$ \\
\hline 6 & $-t-10^{-4}$ & $t-10^{-5}$ & $-\frac{1}{6}-10^{-6}$ & $\frac{1}{b}-10^{-7}$ & $-f-10^{-8}$ & ... \\
\hline$\vdots$ & $\vdots$ & $\vdots$ & $\vdots$ & $\vdots$ & $\vdots$ & \\
\hline
\end{tabular}

Remark 1: Although this method of summing over expanding cubes is not rapidly convergent, it is extremely well behaved. The alternation of the $P(n)$ and $Q(n)$ above and below their limiting values provide precise error bounds, which may be useful in theoretical considerations.

Remark 2: The work of Campbell ${ }^{3}$ must be mentioned at this point. He states general conditions on a doubly indexed series and concludes a convergence result, which is stronger than Theorem 2 above. However, there is a serious error in his proof and his general theorem is false. A simplified version of Campbell's claimed result would be the following: Let $\left\{a_{i j}\right\}_{i=1, j=1}^{\infty}$ be a doubly indexed "sequence" of reals satisfying (A) for all $i,\left\{\left|a_{i 1}\right|,\left|a_{i 2}\right|,\left|a_{i 3}\right|, \ldots\right\}$ is a monotonically decreasing sequence with $\lim _{j \rightarrow \infty} a_{i j}=0$, and for all $j,\left\{\left|a_{1 j}\right|,\left|a_{2 j}\right|, \ldots\right\}$ is a montonically decreasing sequence with $\lim _{i \rightarrow \infty} a_{i j}=0$; and (B) the sign of $a_{i j}$ is $(-1)^{i+j+1}$. Then $\Sigma_{i=1}^{\infty}\left(\Sigma_{j=1}^{\infty} a_{i j}\right)$ exists.

Here is a counterexample to this claim. Let the $a_{i j}$ 's be defined as in the array in Table I. Let

TABLE 1 . The $i j$ th entry in the array is denoted $a_{i j}$, to form a counterexample to the general convergence result claimed by Campbell. 


$$
U_{i}=\sum_{j=1}^{\infty} a_{i j}, \quad \text { for } i=1,2,3, \ldots .
$$

Clearly, each $U_{i}$ exists and the sign of $U_{i}$ is $(-1)^{i+1}$. The odd indexed $U_{i}$ are all positive and easily calculated:

$$
\begin{aligned}
& U_{1}=\ln 2=1 / 1 \times 2+1 / 3 \times 4+1 / 5 \times 6+\cdots, \\
& U_{3}=\ln 2+1=1 / 2 \times 3+1 / 4 \times 5+1 / 6 \times 7+\cdots .
\end{aligned}
$$

In general,

$$
\begin{aligned}
U_{2 k-1} & =(-1)^{k+1}\left[\ln 2-\left(1-\frac{1}{2}+\cdots+(-1)^{k} /(k-1)\right)\right] \\
& =\sum_{j=0}^{\infty} \frac{1}{(k+2 j)(k+2 j+1)}, \quad k \geqslant 2 .
\end{aligned}
$$

Any $U_{2 k}$ is negative and given by

$$
U_{2 k}=\sum_{j=k+1}^{\infty}-\frac{1}{10^{j}}=-\frac{10^{-k}}{9} .
$$

Note, that

$$
\sum_{k=1}^{\infty} U_{2 k}=-\frac{1}{9}\left(\sum_{k=1}^{\infty} 10^{-k}\right)=-\frac{1}{81} .
$$

On the other hand

$$
\begin{aligned}
\sum_{k=1}^{\infty} U_{2 k-1} & =\sum_{k=1}^{\infty}\left(\frac{1}{k(k+1)}+\frac{1}{(k+2)(k+3)}+\cdots\right) \\
& =\sum_{n=1}^{\infty}\left(\frac{1}{n(n+1)}+\frac{1}{(n+1)(n+2)}+\cdots\right) \\
& >\sum_{n=1}^{\infty} \sum_{j=n}^{\infty} \frac{1}{2 j^{2}}>\frac{1}{2} \sum_{n=1}^{\infty} \int_{n}^{\infty} \frac{1}{t^{2}} d t \\
& =\frac{1}{2} \sum_{n=1}^{\infty} \frac{1}{n}=\infty .
\end{aligned}
$$

Since the sum of the positive terms diverge and the sum of the negative terms converge, it follows that $\lim _{n \rightarrow \infty} \Sigma_{i=1}^{n} U_{i}$ does not exist.

Thus,

$$
\sum_{i=1}^{\infty}\left(\sum_{j=1}^{\infty} a_{i j}\right) \text { diverges, }
$$

even though it satisfies Campbell's conditions for convergence. Campbell goes on to claim that the analogous result holds for any dimension and that one could also prove convergence if one summed by expanding rectangles. Both these statements are unfounded.

Remark 3: In light of the above, it appears that there is no simple proof in the literature of the convergence of any of the most elementary direct summation methods. That is why the detailed proofs of Theorems 2 and 4 are given. Emersleben's result (Theorem 3 ) indicates that a noncasual approach is justified.

Remark 4: The proofs given to Theorems 2 and 4 are simple and intuitive, based as they are on the fact that the contribution of a basic unit cell to the sum is always of the sign of the nearest point in the cell to the origin. We have abstracted this property and have obtained quite general convergence results for multidimensional alternating series. These results will be published elsewhere. We will point out later that Theorems 2 and 4 also follow from the deeper considerations of the next section.

\section{INTEGRAL TRANSFORMATIONS AND ANALYTICITY}

Our purpose in this section is to establish a firm connection between the elementary direct summation methods discussed above and the integral transformation methods, which are described by Glasser and Zucker in their survey article. ${ }^{1}$ One major consequence of this connection is that we can give a definition of Madelung's constant, which has a firm mathematical foundation and is unique in a strong enough sense to indicate why diverse methods of performing the lattice sums lead to the same number. We begin with a general discussion of analyticity of certain lattice sums in $\mathrm{N}$ dimensional space. Of course, $N=2$ and 3 are the most interesting cases, but the general notation is just as convenient.

For a complex number $s$, let $\operatorname{Re} s$ denote the real part of $s$ and let

$$
A^{N}(s)=\left\{(-1)^{\mathrm{n}} /\|\mathbf{n}\|^{2 s}: \mathbf{n} \in \mathbb{Z}^{N} /\{0\}\right\},
$$

where for $\mathbf{n}=\left(n_{1}, n_{2}, \cdots, n_{N}\right) \in \mathbb{Z}^{N},(-1)^{\mathbf{n}}=(-1)^{n_{1}+\cdots+n_{N}}$ and $\|\mathbf{n}\|=\left(n_{1}^{2}+n_{2}^{2}+\cdots+n_{N}^{2}\right)^{1 / 2}$. We also use the notations

$$
|\mathbf{n}|=\left(\left|n_{1}\right|,\left|n_{2}\right|, \ldots,\left|n_{N}\right|\right) \in \mathbb{Z}^{N},
$$

and for $m \in \mathbb{Z}^{N}$,

$\mathrm{n} \geqslant \mathbf{m}, \quad$ if $n_{j} \geqslant m_{j}, \quad$ for $1 \leqslant j \leqslant N$.

If $\operatorname{Re} s>N / 2$, then a simple comparison test shows that $\Sigma_{\mathbf{n} \neq 0} 1 / \| \mathbf{n}||^{2 s}<\infty$. So the elements of $A^{N}(s)$ are absolutely summable if $\operatorname{Re} s>N / 2$. Let

$$
d_{N}(2 s)=\sum\left\{\frac{(-1)^{\mathbf{n}}}{\| \mathbf{n}||^{2 s}}: \mathbf{n} \in \mathbb{Z}^{N} /\{\mathbf{0}\}\right\} .
$$

Then $d_{N}(z)$ is a function of the complex variable $z$ for $\operatorname{Re} z>N$. In fact, it is a multidimensional zeta function, analytic on this domain. To see this, define for $m \in Z^{n}$, with $m>0$,

$$
d^{\mathbf{m}}(z)=\sum\left\{\frac{(-1)^{\mathbf{n}}}{\|n\|^{z}}: \mathbf{n} \in Z^{N} /\{0\} \text { and }|\mathbf{n}|<\mathbf{m}\right\} \text {. }
$$

The $d^{m}(z)$ is analytic for $\operatorname{Re} z>0$. For fixed $\delta>0$, if $\operatorname{Re} z>N+\delta$,

$$
\left|d_{N}(z)-d^{\mathbf{m}}(z)\right|<\sum\left\{\frac{1}{\|\left.\mathbf{n}\right|^{N+\delta}}: \mathbf{n} \in \mathbf{Z}^{N} \backslash\left\{\mathbf{l} \in \mathbf{Z}^{N}:|\mathbf{l}|<\mathbf{m}\right\}\right\} .
$$

The right-hand side of (21) can be made arbitrarily small by letting the minimal coefficient of $m$ get large. Thus, on the region $(\operatorname{Re} z>N+\delta), d_{N}(z)$ is the uniform limit of analytic functions and is therefore analytic. Since $\delta>0$ was arbitrary we have established the following proposition.

Proposition 1: The function $d_{N}(2 s)$ is analytic in $s$ for $\operatorname{Re} s>N / 2$.

Now comes the crucial step for the definition of Madelung's constant. The functions $d_{N}(2 s)$ can be analytically continued to the region $(\operatorname{Re} s>0)$. To accomplish this we follow the ideas of Glasser and Zucker ${ }^{1}$ and introduce $\theta$ functions and the Mellin transform. We need, in particular,

$$
\theta_{4}(q)=\sum_{n=-\infty}^{\infty}(-1)^{n} q^{n^{2}}, \quad 0<q<1 .
$$

So 


$$
\theta_{4}\left(e^{-t}\right)=\sum_{n=-\infty}^{\infty}(-1)^{n} e^{-n^{2} t}, \quad 0<t<\infty .
$$

For a continuous function $f(t)$ defined for $0<t<\infty$, bounded as $t \rightarrow 0$ and decaying sufficiently fast as $t \rightarrow \infty$, one can define a normalized Mellin transform $M_{s}(f)$ for $\operatorname{Re} s>0$ by

$$
M_{s}(f)=\Gamma^{-1}(s) \int_{0}^{\infty} f(t) t^{s-1} d t,
$$

where $\Gamma$ is the usual gamma function given by

$$
\Gamma(s)=\int_{0}^{\infty} e^{-t} t^{s-1} d t, \quad \text { for } \operatorname{Re} s>0 .
$$

Of course, $\Gamma$ and $\Gamma^{-1}$ are analytic functions on $(\operatorname{Re} s>0)$. A useful property of the Mellin transform is that for $a>0$ and $f$ such that its Mellin transform exists, $M_{s}\left(\tau_{a} f\right)=M_{s}(f) / a^{s}$, where $\tau_{a} f(t)=f(a t)$ for all $t>0$. In particular,

$$
M_{s}\left(e^{-a t}\right)=1 / a^{s}, \quad \text { for } a>0 .
$$

Consider now a truncation of the series for $\theta_{4}$. For some positive integer $m$, let $\phi_{m}(q)=\Sigma_{n=-m}^{m}(-1)^{n} q^{n^{2}}$. If $\mathbf{m} \in \mathbf{N}^{N}$, say $\mathbf{m}=\left(m_{1}, \ldots, m_{N}\right)$, then let $m=\min \left\{m_{1}, \ldots m_{N}\right\}$. We wish to approximate the $N$ th power of $\theta_{4}$ with products of $\phi_{m_{i}}$. For $0<t<\infty$,

$$
\begin{gathered}
\left|\theta_{4}^{N}\left(e^{-t}\right)-\prod_{i=1}^{N} \phi_{m_{i}}\left(e^{-t}\right)\right| \\
<\mid \prod_{i=1}^{N}\left[\left|\theta_{4}\left(e^{-t}\right)-\phi_{m_{i}}\left(e^{-t}\right)\right|\right. \\
\left.\quad+\phi_{m_{i}}\left(e^{-t}\right)\right]-\prod_{i=1}^{N} \phi_{m_{i}}\left(e^{-t}\right) \mid \\
=\prod_{i=1}^{N}\left(b_{i}+a_{i}\right)-\prod_{i=1}^{N} a_{i},
\end{gathered}
$$

where $a_{i}=\phi_{m_{i}}\left(e^{-t}\right)$ and $b_{i}=\left|\theta_{4}\left(e^{-t}\right)-\phi_{m_{i}}\left(e^{-t}\right)\right|$. Note that $0<a_{i}, b_{i}<1$, for $i=1, \ldots, N$ and the last expression in (24), $\Pi_{i=1}^{N}\left(b_{i}+a_{i}\right)-\Pi_{i=1}^{N} a_{i}$ represents the difference in volume between an $N$ box of side lengths $b_{i}+a_{i}, i=1, \ldots, N$ and one of side lengths $a_{i}, i=1, \ldots, N$. Clearly $\Pi_{i=1}^{N}\left(b_{i}+a_{i}\right)$ $-\Pi_{i=1}^{N} a_{i}<\max \left\{b_{i}: 1<i<N\right\} N 2^{N-1}$. Now $\mid \theta_{4}\left(e^{-t}\right)$ $-\phi_{m}\left(e^{-t}\right) \mid$ is the maximum $b_{i}$ and $\mid \theta_{4}\left(e^{-t}\right)$ $-\phi_{m}\left(e^{t}\right) \mid<2 e^{-m^{2} t}$. So (24) becomes

$$
\left|\theta_{4}^{N}\left(e^{-t}\right)-\prod_{i=1}^{N} \phi_{m_{i}}\left(e^{-t}\right)\right|<N 2^{N} e^{-m^{2} t} .
$$

We also need the Mellin transform of $\Pi_{i=1}^{N} \phi_{m_{i}}\left(e^{-t}\right)-1$, which is easily found using linearity and (23):

$$
\begin{aligned}
M_{s}\left[\prod_{i=1}^{N} \phi_{m_{i}}\left(e^{-t}\right)-1\right] & =M_{s}\left[\sum_{|\mathbf{n}|<\mathbf{m}}^{\prime}(-1)^{\mathbf{m}} e^{-|| \mathbf{n}||^{2 t}}\right] \\
& =\sum_{|\mathbf{m}|<\mathbf{m}}^{\prime} \frac{(-1)^{\mathbf{n}}}{\|\left.\mathbf{n}\right|^{2 s}} \\
& =d^{\mathbf{m}}(2 s) .
\end{aligned}
$$

The prime on the summation sign indicates that the $\mathbf{n}=\mathbf{0}$ term is omitted.

We are now ready for the main theorems of this section. Define $F(s)=M_{s}\left[\theta_{4}^{N}\left(e^{-t}\right)-1\right]$ wherever it exists.
Theorem 5: The Mellin transform $F(s)$ of $\theta_{4}^{N}\left(e^{-t}\right)-1$ exists and is analytic for all $s$ with $\operatorname{Re} s>0$. Furthermore $F$ provides an analytic continuation of $d_{N}(2 s)$ to the region $(\operatorname{Re} s>0)$.

Theorem 6: For any $m \in \mathbb{Z}^{N}, \mathrm{~m} \geq 0, m=\min \left(m_{i}\right.$ : $1<i<N\}$ and $\operatorname{Re} s>0$

$$
\left|F(s)-d^{m}(2 s)\right|<N 2^{N} \Gamma(\operatorname{Re} s) /\left(m^{2 \operatorname{Re} s}|\Gamma(s)|\right) .
$$

Proof (of Theorems 5 and 6 combined): Let $s$ be a complex number such that $\operatorname{Re} s>0$. Since

$0<1-\theta_{4}^{N}\left(e^{-t}\right)<N\left[1-\theta_{4}\left(e^{-t}\right)\right]<N e^{-t}$,

for all $0<t<\infty$,

then

$$
\begin{aligned}
& \int_{0}^{\infty}\left|\theta_{4}^{N}\left(e^{-t}\right)-1\right|\left|t^{s-1}\right| d t \\
& \quad<N \int_{0}^{\infty} e^{-t} t^{\operatorname{Re} s-1} d t=N \Gamma(\operatorname{Re} s) .
\end{aligned}
$$

Therefore, if $\operatorname{Re} s>0$, then

$$
\int_{0}^{\infty}\left[\theta_{4}^{N}\left(e^{-t}\right)-1\right] t^{s-1} d t=F(s)
$$

exists. Using (25) and (26), with $m$ as in Theorem 6 ,

$$
\begin{aligned}
|\Gamma(s)| & \left|F(s)-d^{\mathrm{m}}(2 s)\right| \\
& \leqslant \int_{0}^{\infty}\left|\theta_{4}^{N}\left(e^{-t}\right)-\prod_{i=1}^{N} \phi_{m_{l}}\left(e^{-t}\right)\right|\left|t^{s-1}\right| d t \\
& \leqslant N 2^{N} \int_{0}^{\infty} e^{-m^{2} t} t^{\operatorname{Re} s-1} d t \\
& =N 2^{N} \Gamma(\operatorname{Re} s) / m^{2} \text { Res. }
\end{aligned}
$$

Thus (27) holds. In turn, (27) implies that $F(s)$ can be uniformly approximated by the $d^{\mathrm{m}}$ (2s) on any region of the form $R_{\delta, M}=\{s:|s|<M$ and $\operatorname{Re} s>\delta\}$. To see this let $K$ be an upper bound for the continuous function $N 2^{N}(\operatorname{Re} s) /|\Gamma(s)|$ on the closure of $R_{\delta, M}$. Then, for any $\epsilon>0$ and any $m$ such that $m=\min \left\{m_{1}, \ldots, m_{N}\right\}>(K / \epsilon)^{1 / 2 \delta}$,

$$
\left|F(s)-d^{\mathrm{m}}(2 s)\right|<\epsilon, \text { for all } s \in R_{\delta, M} \text {. }
$$

Since $\epsilon$ is arbitrary and $\mathrm{d}^{\mathrm{m}}$ is analytic, $F$ is analytic on $R_{\delta, M}$ for any $\delta>0$ and $0<M<\infty$. Therefore $F$ is analytic on $(\operatorname{Re} s>0)$. Finally, it is now clear that $F(s)$ agrees with $d_{N}(2 s)$ if $\operatorname{Re} s>N / 2$. Thus $F$ is an analytic continuation of $d_{N}$.

Q.E.D.

In light of Theorem 5, we will drop the use of $F$ and write

$$
d_{N}(2 s)=M_{s}\left[\theta_{4}^{N}\left(e^{-t}\right)-1\right], \quad \text { for } \operatorname{Re} s>0 .
$$

A rigorous mathematical definition can now be given for Madelung's constant.

Definition: For a three-dimensional NaCl-type ionic crystal, Madelung's constant is the number

$$
d_{3}(1)=M_{1 / 2}\left[\theta_{4}^{3}\left(e^{-t}\right)-1\right] \text {. }
$$

Of course, this is the very number that has been approximated by many different methods over the years. We have just given a definition that avoids all the ambiguities of meaning that have existed. The uniqueness of analytic continuation explains the special significance of this particular sum of elements of 


$$
\left\{(-1)^{i+j+k} /\left(i^{2}+j^{2}+k^{2}\right)^{1 / 2}:(i, j, k) \in \mathbf{Z}^{3} /(0,0,0)\right\} .
$$

Formula (27) emphasizes the strong connection between the integral transformation methods and the direct summation methods. In fact it is worthwhile to formulate a corollary to Theorem 6, which gives explicit error bounds for a finite sum approximation to Madelung's constant.

Corollary 1: Let $m_{i}>0$, for $i=1,2,3$ and $m=\min \left\{m_{1}, m_{2}, m_{3}\right\}$. Then

$$
\left|d_{3}(1)-\sum_{|| l|,| j|,| k \mid)<m}^{\prime} \frac{(-1)^{i+j+k}}{\left(i^{2}+j^{2}+k^{2}\right)^{1 / 2}}\right|<\frac{12}{m} .
$$

Remark 5: The above corollary says the Madelung's constant for $\mathrm{NaCl}$ can be obtained, not only by expanding cubes, but by expanding any rectilinear shape and the order of convergence is the inverse of the minimum dimension. In fact, it is permissible to let some coordinates go to infinity before others.

Remark 6: Of course Theorems 2 and 4 follow immediately from Theorems 5 and 6 but we preferred to present the simple direct proofs of Secs. II and III for the reasons given in remark 4.

\section{BACK TO TWO DIMENSIONS}

In this section we consider the analyticity of various methods of summing the elements of the set

$$
A_{s}=\left\{(-1)^{j+k} /\left(j^{2}+k^{2}\right)^{s}:(j, k) \in \mathbf{Z} /(0,0)\right\} .
$$

From Theorem 6, it follows that the method of expanding squares leads to $d_{2}(2 s)$, which is analytic for $\operatorname{Re} s>0$. In fact, expanding rectangles of any shape with sides parallel to the axes lead to $d_{2}(2 s)$. In Theorem 1, we showed that the method of expanding circles converged when $s=\frac{1}{2}$, but there is no reason to believe that $d_{2}(1)$ is obtained unless one shows that the appropriate function is analytic. Using the notation of Sec. II, let

$$
G(s)=\sum_{n=1}^{\infty} \frac{(-1)^{n} C_{2}(n)}{n^{s}},
$$

whenever the right-hand side converges. Then $G(s)$ is the sum of the elements of $A_{s}$ obtained by expanding circles.

Theorem 7: The function $G(s)$ exists and is analytic for $\operatorname{Re} s>\frac{1}{3}$. Thus, $G(s)=d_{2}(2 s)$ if $\operatorname{Re} s>\frac{1}{3}$; in particular, $d_{2}(1)=\Sigma_{n=1}^{\infty}(-1)^{n} C_{2}(n) / n^{1 / 2}$.

Proof: As in the proof of Theorem 1, let $B_{n}$ $=\Sigma_{k=1}^{n}(-1)^{k} C_{2}(k)$. By (8),

$$
B_{n}=O\left(n^{1 / 3}-\tau\right) \text {, for some } \epsilon>0 \text {. }
$$

Define $G_{n}(s)$ for all $s$ with $\operatorname{Re} s>0$ by

$$
G_{n}(s)=\sum_{k=1}^{n} \frac{(-1)^{k} C_{2}(k)}{k^{s}}
$$

As in (9)

$$
G_{n}(s)=\frac{B_{n}}{(n+1)^{s}}+\sum_{k=1}^{n} B_{k}\left[k^{-s}-(k+1)^{-s}\right] .
$$

By (30), if $\operatorname{Re} s>1$, then $\left|B_{n} /(n+1)^{s}\right| \rightarrow 0$, uniformly in $s$. Note that

$$
\begin{aligned}
\mid k^{-s} & -(k+1)^{s} \mid \\
& =\left|(-s) \int_{k}^{k+1} t-(s+1) d t\right|<|s| \int_{k}^{k+1} t-(\operatorname{Re} s+1) d t \\
& \leqslant|s| k-(\operatorname{Res}+1) .
\end{aligned}
$$

So for $s \in R_{M}=\left\{z: \operatorname{Re} z>\frac{1}{3},|z|<M\right\}$ with $M$ a fixed positive number and $1 \leqslant N \leqslant N^{\prime}$,

$$
\begin{aligned}
\left|\sum_{k=N}^{N^{\prime}} B_{k}\left[k^{-s}-(k+1)^{-s}\right]\right| & \\
& <\sum_{k=N}^{N^{\prime}}\left|B_{k}\right|\left|k^{-s}-(k+1)^{-s}\right| \\
& <K \sum_{k=N}^{N^{\prime}} k^{1 / 3-\epsilon}\left|k^{-s}-(k+1)^{-s}\right| \text { by }(30) \\
& <K M \sum_{k=N}^{N^{\prime}} k^{1 / 3-\epsilon-\operatorname{Res}-1} \text { by (32) } \\
& <K M N^{1 / 3-\epsilon-\operatorname{Res}} \\
& <K M N^{-\epsilon} \\
& \rightarrow 0 \text { as } N, N^{\prime} \rightarrow \infty,
\end{aligned}
$$

uniformly for $s \in R_{m}$. Thus, the sequence of functions $\left\{G_{n}(s)\right\}_{n=1}^{\infty}$ is uniformly Cauchy on $R_{M}$ and it converges uniformly to a limit function $G(s)$. Furthermore, each $G_{n}(s)$ is analytic, so $G(s)$ is analytic for $s \in R_{M}$. Since $M$ is arbitrary, $G(s)$ exists and is analytic for all $s$ with $\operatorname{Re} s>\frac{1}{3}$. Q.E.D.

Remark 7: It is not know what the minimum non-negative $\beta$ is, such that $G(s)$ exists for all $s$ with $\operatorname{Re} s>\beta$. However, if we consider another method of summing the elements of $A_{s}$, we can get a very complete and illuminating analysis. This is the method of expanding diamonds.

For each $k=1,2,3, \ldots$, and complex $s$ with $\operatorname{Re} s>0$, let

$$
\delta_{k}(s)=\sum_{j=0}^{k}\left\{(k-j)^{2}+j^{2}\right\}^{-s} .
$$

For each $n=1,2,3, \ldots$, let

$$
\Delta_{n}(s)=4 \sum_{k=1}^{n}(-1)^{k} \delta_{k}(s)-4 X_{n}(s),
$$

where $X_{n}(s)=\Sigma_{k=1}^{n}(-1)^{k} / k^{s}$. Note that $\Delta_{n}(s)$ counts the contributions within the diamond $|k|+|j|<n$. Now, $\lim _{n \rightarrow \infty} X_{n}(s)=\Sigma_{k=1}^{\infty}(-1)^{k} / k^{s}=-\eta(s)$ and $\eta$ is known to be analytic for $\operatorname{Re} s>0$. Therefore, in order to determine for which $s$ the limit of the $\Delta_{n}(s)$ exists and is analytic, it is sufficient to analyze $\Sigma_{k=1}^{\infty}(-1)^{k} \delta_{k}(s)$. We begin by establishing a number of facts about the sequence of $\delta_{k}$.

Proposition 2: (a) $\lim _{k \rightarrow \infty} \delta_{k}\left(\frac{1}{2}\right)=\sqrt{2} \ln (\sqrt{2}+1)$. Thus $\Sigma_{k=1}^{\infty}(-1)^{k} \delta_{k}\left(\frac{1}{2}\right)$ diverges.

(b) For real $r>\frac{1}{2}, \delta_{k-1}(r)>\delta_{k}(r), k=2,3,4, \ldots$.

(c) $\Sigma_{k=1}^{\infty}(-1)^{k} \delta_{k}(s)$ exists and is analytic for $\operatorname{Re} s>\frac{1}{2}$.

Proof:(a)

$$
\begin{aligned}
\delta_{k}\left(\frac{1}{2}\right) & =\sum_{j=0}^{k}\left[(k-j)^{2}+j^{2}\right]^{-1 / 2} \\
& =\sum_{j=0}^{k}\left(\frac{1}{k}\right)\left[\left(1-\frac{j}{k}\right)^{2}+\left(\frac{j}{k}\right)^{2}\right]^{-1 / 2} \\
& \rightarrow \int_{0}^{1}\left[(1-t)^{2}+t^{2}\right]^{-1 / 2} d t, \quad \text { as } k \rightarrow \infty \\
& =\sqrt{2} \ln (\sqrt{2}+1) .
\end{aligned}
$$


For the proofs of $(b)$ and $(c)$ it is very convenient to introduce the following function. For $\operatorname{Re} s>\frac{1}{2}$, let

$$
V(s)=2 s\left(2^{s}\right) \int_{0}^{\pi / 4} \cos ^{2 s} \theta d \theta-1 .
$$

Then $V$ is continuous and $V\left(\frac{1}{2}\right)=0$. If $r \geq \frac{1}{2}$, then

$$
\begin{aligned}
V(r) & =2 r \int_{0}^{\pi / 4}\left(2 \cos ^{2} \theta\right)^{r} d \theta-1 \\
& >\int_{0}^{\pi / 4} \sqrt{2} \cos \theta d \theta-1=0 .
\end{aligned}
$$

We proceed now to the proof of $(b)$.

Let $r>1$ and $k>2$,

$$
\begin{aligned}
\delta_{k-1}(r)-\delta_{k}(r) \\
=\sum_{j=1}^{k}\left\{\left[(k-j)^{2}+(j-1)^{2}\right]^{-r}-\left[(k-j)^{2}+j^{2}\right]^{-r}\right\}-k^{-2 r} \\
=\sum_{j=1}^{k} \int_{j-1}^{j} \frac{2 r t d t}{\left[(k-j)^{2}+t^{2}\right]^{r+1}}-k^{-2 r}>\sum_{j=1}^{k} \int_{j-1}^{j} \frac{2 r t d t}{\left[(k-t)^{2}+t^{2}\right]^{r+1}}-k^{-2 r} \\
=\int_{0}^{k} \frac{2 r t d t}{\left[(k-t)^{2}+t^{2}\right]^{r+1}}-k^{-2 r}=k^{-2 r}\left\{\int_{0}^{1} \frac{2 r u d u}{\left[(1-u)^{2}+u^{2}\right]^{r+1}}-1\right\} \\
=k^{-2 r}\left\{2 r \int_{-1 / 2}^{1 / 2} \frac{(v+1 / 2) d v}{2^{r+1}\left(v^{2}+1 / 4\right)^{r+1}}-1\right\} \quad\left(v=u-\frac{1}{2}\right) \\
=k^{-2 r}\left\{\frac{2 r}{2^{r+1}} \int_{0}^{1 / 2} \frac{d v}{\left(v^{2}+1 / 4\right)^{r+1}}-1\right\}=k^{-2 r}\left[2 r \int_{0}^{\pi / 4}\left(2 \cos ^{2} \theta\right)^{r} d \theta-1\right\} \quad(\tan \theta=2 v) \\
=k^{-2 r V(r)>0 .}
\end{aligned}
$$

That is, $\delta_{k-1}(r)>\delta_{k}(r)$, for $r \geq \frac{1}{2}, k=2,3,4, \ldots$.

To prove (c), let $\epsilon>0$ and $M<\infty$ be arbitrary. Let

$$
R=\left\{z: \operatorname{Re} z>\frac{1}{2}+\epsilon \text { and }|z|<M\right\} .
$$

For $s \in R$, let $r=\operatorname{Re} s$. For $k>2$, we can write

$$
\begin{aligned}
\delta_{k-1}(s)-\delta_{k}(s) & =\sum_{j=1}^{k-1}\left\{\left[(k-j)^{2}+(j-1)^{2}\right]^{-s}-\left[(k-j)^{2}+j^{2}\right]^{-s}\right\}+(k-1)^{-2 s}-2 k-2 s \\
& =\sum_{j=1}^{k-1} \int_{j-1}^{j} \frac{2 s t d t}{\left[(k-j)^{2}+t^{2}\right]^{s+1}}+(k-1)^{-2 s}-2 k-2 s .
\end{aligned}
$$

Thus,

$$
\begin{aligned}
\left|\delta_{k-1}(s)-\delta_{k}(s)\right| & <\sum_{j=1}^{k-1} \int_{j-1}^{j} \frac{2|s| t d t}{\left[(k-j)^{2}+t^{2}\right]^{r+1}}+3(k-1)^{-2 r} \\
& <2 M \sum_{j=1}^{k-1} \int_{j-1}^{j} \frac{t d t}{\left[(k-1-t)^{2}+t^{2}\right]^{r+1}}+3(k-1)^{-2 r} \\
& =2 M \int_{0}^{k-1} \frac{t d t}{\left[(k-1-t)^{2}+t^{2}\right]^{r+1}}+3(k-1)^{-2 r} \\
& =(k-1)^{-2 r}\left\{2 M \int_{0}^{1} \frac{u d u}{\left[(1-u)^{2}+u^{2}\right]^{r+1}}+3\right\}=(k-1)^{-2 r}\{(M / r) V(r)+(M / r)+3\}<(k-1)^{-2 r} C,
\end{aligned}
$$

where $C$ is the maximum of the continuous function $(M /$ $r) V(r)+M / r+3$ for $\frac{1}{2}+\epsilon<r<M$. Now, for each $n$, $\sum_{k=1}^{n}\left(\delta_{2 k-1}(s)-\delta_{2 k}(s)\right)$ is an analytic function of $s$ for $\operatorname{Re} s>\frac{1}{2}$ and

$$
\sum_{k=1}^{\infty}(-1)^{k} \delta_{k}(s)=\lim _{n \rightarrow \infty}\left[-\sum_{k=1}^{n}\left(\delta_{2 k-1}(s)-\delta_{2 k}(s)\right)\right]
$$

exists uniformly on $R$, by (35) and the Weierstrass $M$ test. Since $\epsilon>0$ and $M<\infty$ are arbitrary, (c) has been established.

Q.E.D.

We can now describe the behavior of the diamond sums.

Theorem 8: For each complex number $s$ with $\operatorname{Re} s>0$ and each $n=1,2, .$. let

$$
\Delta_{n}(s)=\sum_{i=1}^{n}(-1)^{t}\left\{\sum_{|l|+|k|=l}\left[j^{2}+k^{2}\right]^{-s}\right\} .
$$

Then $\lim _{n \rightarrow \infty} \Delta_{n}(s)$ exists and is analytic for $\operatorname{Re} s>\frac{1}{2}$. Although $\left\{\Delta_{n}\left(\frac{1}{2}\right)\right\}_{n=1}^{\infty}$ fails to converge,

$$
d_{2}(1)=\lim _{r \rightarrow 1 / 2+}\left(\lim _{n \rightarrow \infty} \Delta_{n}(r)\right)
$$

Proof: These claims all follow immediately from Proposition 2.

Remark 8: Further analysis along the lines of Proposition 2 shows that although

$$
\sum_{l=1}^{\infty}(-1)^{l}\left\{\sum_{|V|+|k|=l}\left[j^{2}+k^{2}\right]^{-1 / 2}\right\}
$$

is divergent, it is Cesáro summable or Abel summable to $d_{2}(1)$. 
The diamond sums provide a nice illustration of how a method of summing the elements of $A_{s}$ can be analytic in $s$ for $\operatorname{Re} s$ large, then with decreasing $\operatorname{Re} s$, this analyticity fails at a specific point. With the diamond sums it happens to be at $\frac{1}{2}$, with expanding squares or rectangles it is at 0 . It is not clear where the expanding circles fails; it is at some point less than $\frac{1}{3}$. In three dimensions the method of expanding spheres fails at some point greater then $\frac{1}{2}$.

\section{THE HEXAGONAL LATTICE}

As an illustration of what is obtained when one studies other crystal lattices in the above manner, we include a brief summary of results on Madelung's constant of a two-dimensional regular hexagonal lattice with ions of alternating unit charge.

In order to obtain a tractable expression for the terms appearing in the lattice sum, choose a coordinate system with an angle of $\phi=\pi / 3$ between the positive axes. Then an arbitrary site in the lattice has coordinates $(\overline{n, m})$ with $n$ and $m$ integers. A charge of $+1,-1$, or 0 is attached to that site in a regular fashion (see Fig. 2). By considering the two parallelograms indicated in Fig. 2, one can see that this charge may be expressed by

$$
\begin{aligned}
q(n, m)= & \frac{4}{3}[-\sin (n \theta) \sin ((m-1) \theta) \\
& +\sin ((m+1) \theta) \sin ((n+1) \theta)], \quad \theta=2 \pi / 3 .
\end{aligned}
$$
by

The distance of the point $(\overline{n, m})$ from the origin is given

$$
|(\overline{n, m})|=\left[(n+m / 2)^{2}+3(m / 2)^{2}\right]^{1 / 2} .
$$

The set of numbers to be summed is then

$$
\begin{aligned}
C_{s}= & \left\{q(n, m) /|(\overline{n, m})|^{2 s}: \quad(n, m) \in \mathbf{Z}^{2} /(0,0)\right\}, \\
& \text { for } \operatorname{Re} s>0 .
\end{aligned}
$$

As before, the elements of $C_{s}$ are absolutely summable for $\operatorname{Re} s>1$ and we wish an analytic continuation of their sum to a region which includes $s=\frac{1}{2}$. Arguments, like those used for the diamond sums, will show that direct summation by expanding shells of hexagons will converge analytically for $\operatorname{Re} s>\frac{1}{2}$ and even have a limit as $s$ approaches $\frac{1}{2}$ from the right. However, for precise calculation purposes an analytic continuation via the integral transform methods is far superior. Let

$$
H_{2}(2 s)=\sum\left\{\frac{q(n, m)}{\|\left.(\overline{n, m})\right|^{2 s}}:(n, m) \in \mathbf{Z}^{2} /(0,0)\right\},
$$

for $\operatorname{Re} s>1$. Then $H_{2}$ is an analytic function of $s$ and the series converges absolutely. Substituting the expression for $q(n, m)$ and using elementary trignometric identities yields

$$
\begin{aligned}
H_{2}(2 s)= & \frac{2}{3} \sum^{\prime} \frac{\cos ((m-n) \theta)}{|(\overline{n, m})|^{2 s}} \\
& -\left[\frac{1}{3} \sum^{\prime} \frac{\sin ((m-n) \theta)}{|(\overline{n, m})|^{2 s}}\right],
\end{aligned}
$$

where $\Sigma^{\prime}$ indicates the sum is over $(n, m) \in \mathbb{Z}^{2} /(0,0)$. By symmetry considerations, the second term in the right-hand side of (38) is zero. Further manipulation of theta functions (using the modular equation of order 3) produces a rectangular sum

$$
\begin{aligned}
H_{2}(2 s)= & \left(1-3^{1-s}\right)\left[\sum^{\prime} \frac{1}{\left(n^{2}+3 m^{2}\right)^{s}}\right. \\
& \left.-\frac{1}{2} \sum^{\prime} \frac{(-1)^{n+m}}{\left(n^{2}+3 m^{2}\right)^{s}}\right] .
\end{aligned}
$$

A theta function identity due to Cauchy ${ }^{10}$ and a Mellin transform yields

$$
H_{2}(2 s)=3\left(1-3^{1-s}\right) \xi(s) L_{-3}(s),
$$

where $\xi(s)$ is the standard zeta function $\left(\sum_{n=1}^{\infty} n^{-s}\right)$ and

$$
L_{-3}(s)=1-2^{-s}+4^{-s}-5^{-s}+7^{-s}-8^{-s}+\cdots \text {. }
$$

The formula (40) can also be deduced directly from (38) by using results in Sec. IV of Glasser and Zucker. ${ }^{1}$ While the intermediate sums (39) and (40) are only analytic for $\operatorname{Re} s>1$, the standard continuation of the zeta function,

$$
\left(1-2^{1-s}\right) \xi(s)=\sum_{n=1}^{\infty}(-1)^{n+1} n^{-s}=\alpha(s)
$$

gives

$$
H_{2}(2 s)=3\left(1-3^{1-s}\right)\left(1-2^{1-s}\right)^{-1} \alpha(s) L_{-3}(s) .
$$

The right-hand side of (41) is an analytic function of $s$ for $\operatorname{Re} s>0$ and therefore (41) provides the required analytic continuation, which is necessary for Madelung's constant of this hexagonal crystal lattice:

$$
H_{2}(1)=3(\sqrt{3}-1)(\sqrt{2}+1) \alpha(1 / 2) L_{-3}(1 / 2) .
$$

This can be considered as a solution to this lattice sum problem, as both $\alpha\left(\frac{1}{2}\right)$ and $L_{-3}\left(\frac{1}{2}\right)$ can be rapidly calculated by known techniques. At $s=1$, we have an exact result:

$$
H_{2}(2)=\sqrt{3} \pi \log 3 \text {. }
$$

\section{CONCLUDING REMARKS}

We have investigated some fundamental properties of the multiply indexed series involved in the definition of Madelung's constant for an $\mathrm{NaCl}$-type ionic crystal in two and three dimensions. We have provided elementary proofs that convergent series are obtained if the series is summed by letting the shape of a basic unit cell expand. The natural method of summing the effects of all ions within a fixed distance and letting the distance go to infinity leads to a convergent series in two dimensions but not so in three dimensions.

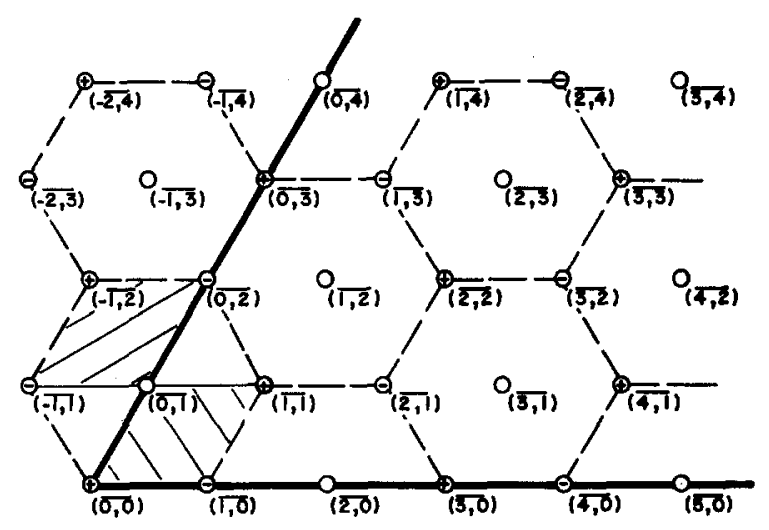

FIG. 2. The hexagonal lattice. 
We have provided a unity to the concept of Madelung's constant by the use of analytic continuation of a complex function. Thus, although conditionally convergent when summed by expanding squares (or cubes), other methods of summing will provide the same answer provided that they are "analytic" in the correct sense. We have provided an analysis of the expanding circles and expanding diamonds methods in two dimensions to illustrate this point.

Perhaps the most important results are those in Sec. IV, rationalizing the integral transformation methods with the direct summation methods. These integral transform methods are the most useful in practice as they lead to very rapidly convergent series.

In the course of these investigations we have encountered many curious facts, most of which are probably known to experts in the area. However, the formulas (42) and (43) seem to be unknown and may be of sufficient interest to have been included; at least, as an illustration that the techniques of analytic continuation are applicable to other lattices.

\section{ACKNOWLEDGMENTS}

The authors would like to thank Professor O. Knop for originally bringing our attention to the problems involved in lattice sums.

The Natural Sciences and Engineering Research Council of Canada partially supported this work.

${ }^{1}$ M. L. Glasser and I. J. Zucker, in Theoretical Chemistry: Advances and Perspectives, edited by D. Henderson (Academic, New York, 1980), Vol. 5, pp. 67-139.

${ }^{2}$ E. L. Burrows and S. F. A. Kettle, J. Chem. Ed. 52, 58 (1975).

${ }^{3}$ E. S. Campbell, J. Phys. Chem. Solids 24, 197 (1963).

${ }^{4}$ H. Evjen, Phys. Rev. 39, 675 (1932).

${ }^{5}$ K. Højendahl, K. Dan. Vidensk. Selsk. Mat. Fys. Medd. 16, 135 (1938).

${ }^{6}$ P. Ewald, Ann. Phys. 64, 253 (1921).

${ }^{7}$ C. Kittel, Introduction To Solid State Physics (Wiley, New York, 1953).

${ }^{8}$ J. S. Blakemore, Solid State Physics (Saunders, Philadelphia, 1969).

${ }^{9}$ O. Emersleben, Math. Nachr. 4, 468 (1951).

${ }^{10}$ L. F. Dickson, Theory of Numbers (Chelsea, New York, 1920), Vol. II.

"G. H. Hardy and E. M. Wright, An Introduction to the Theory of Numbers, $4^{*}$ (Clarendon, Oxford, 1960). 